\title{
On the Stability of the Two-sector Neoclassical Growth Model with Externalities*
}

\author{
Berthold Herrendorf \\ Arizona State University \\ Ákos Valentinyi \\ University of Southampton; Institute of Economics of the \\ Hungarian Academy of Sciences; CEPR
}

January 2005

\begin{abstract}
We study a class of two-sector neoclassical growth models, in which one sector produces consumption goods and the other sector produces the capital goods for both sectors and in which the capital-producing sector has sector-specific externalities. We show analytically that if the capital goods for the two sectors are imperfect substitutes, then local indeterminacy near the steady state is impossible for every empirically plausible specification of the model parameters. More specifically, we show that a necessary condition for local indeterminacy is an upward-sloping aggregate labor demand curve in the capital sector, which requires a counterfactual strength of the externality. We show numerically that an elasticity of substitution of plausible size implies determinacy near the steady state for all empirically plausible specifications of the model parameters. These findings differ sharply from the standard result that if the two capital goods are perfect substitutes, then local indeterminacy occurs in the two-sector model for a wide range of plausible parameter values.

Keywords: Imperfect Substitutability; Sector-specific Externality; Determinacy; Local Indeterminacy.

JEL classification: E0; E3.
\end{abstract}

${ }^{*}$ We are particularly indebted to Manuel Santos for his help. Moreover, we have profited from the comments of Jess Benhabib, Robin Mason, Salvador Ortigueira, Juuso Välimäki, Mark Weder, the audiences at Carlos III, Frankfurt, the Helsinki School of Economics, and Southampton. Herrendorf acknowledges research funding from the Spanish Dirección General de Investigación (Grant BEC2000-0170), from the European Union (Project 721 "New Approaches in the Study of Economic Fluctuations”), and from the Instituto Flores de Lemus (Universidad Carlos III de Madrid). 


\section{Introduction}

We study the local stability properties of the neoclassical growth model at the steady state. Local stability analysis provides important information about the local uniqueness of equilibrium at the steady state and about the way in which business cycles can occur in the model economy. If the steady state is saddle-path stable, then equilibrium is locally unique at the steady state. This is called determinacy and it implies that business cycles must come from shocks to the fundamentals of the model (typically technology shocks). In contrast, if the steady state is stable, then a continuum of equilibrium paths converge to the steady state, implying a severe form of local non-uniqueness of equilibrium. This is called local indeterminacy and it implies that business cycles can come from self-fulfilling shocks to individual beliefs. Since both determinacy and local indeterminacy are theoretically possible when there is some form of non-convexity, we ask which one prevails for empirically plausible choices of parameter values.

We restrict our attention to a class of two-sector neoclassical growth models with sector-specific, positive externalities. In these models, one sector produces a consumption good and the other sector produces the capital goods for the two sectors. We therefore call these sectors the consumption sector and the capital sector. The class of models we consider has been the focus of the recent research on local indeterminacy. ${ }^{1}$ The main finding of this research is that local indeterminacy can occur for mild, empirically plausible externalities in the capital sector, which are consistent with a downward-sloping aggregate labor demand curve. In contrast, in the class of standard one-sector neoclassical growth models, local indeterminacy requires strong externalities that make the aggregate labor demand curve upward sloping [Benhabib and Farmer (1994) and Farmer and Guo (1994)]. Such strong externalities are empirically implausible and an upward-sloping aggregate labor demand curve has awkward economic implications [Aiyagari (1995)].

Our main finding is that local indeterminacy depends critically on whether or not the capital good

\footnotetext{
${ }^{1}$ Examples include Boldrin and Rustichini (1994), Benhabib and Farmer (1996), Benhabib and Nishimura (1998), Perli (1998), Weder (1998), Benhabib et al. (2000), Weder (2000), Schmitt-Grohé (2000), Harrison (2001), and Harrison and Weder (2001). Benhabib and Farmer (1999) provide a review.
} 
for the consumption sector and the capital good for the capital sector are perfect substitutes. Perfect substitutability means that the two new capital goods are perfect substitutes and that the two installed capital goods are perfect substitutes. The common assumption of the literature on indeterminacy is that this is the case. Perfect substitutability implies that the production possibility frontier of the capital sector is linear and installed capital is not sector specific ("putty-putty"). Imperfect substitutability means that the two new capital goods are imperfect substitutes and that the two installed capital goods are imperfect substitutes. This is the empirically plausible case; see for example Huffman and Wynne (1999). Imperfect substitutability implies that the production possibility frontier is strictly concave and installed capital is sector specific ("putty-clay"). We show that imperfect substitutability has two implications. First, we show analytically that local indeterminacy does not occur if the two capital goods are imperfect substitutes and the aggregate labor demand curve slopes downward. Surprisingly, this holds true whenever substitutability is imperfect no matter what the elasticity of substitution is. This result differs sharply from that of the literature, which assumes perfect substitutability and finds that local indeterminacy can easily occur if the aggregate labor demand curve slopes downward. Second, we show numerically that equilibrium is determinate (instead of unstable) for empirically plausible values of the elasticity of substitution of the two capital goods and of the externality. This result is robust to reasonable changes in the parameter values.

Imperfect substitutability of the different capital goods can be due to capital adjustment costs. In Herrendorf and Valentinyi (2003), we explore the local stability properties of the two-sector model with the standard capital adjustment costs used by Lucas and Prescott (1971). We show that it matters whether the adjustment costs apply to the total capital stock of the economy or to each sector's capital stock: local indeterminacy is easier to obtain in the first case than in the second case. All results of Herrendorf and Valentinyi (2003) are numerical. The present paper goes beyond it in three aspects. First, the key result of the present paper is analytical. Second, the key result of the present paper is stronger: given a downward-sloping aggregate labor demand curve (which is the empirically plausible case), we show here that local indeterminacy does not occur for any positive elasticity of substitution between the different capital goods irrespective of where it comes from. In contrast, previously we 
have shown only that it does not occur for some typical parameter choices. Third, the present paper provides the economic intuition for the effects of imperfect substitutability, which was missing from our previous paper.

The intuition follows from the relationship between the relative price of the two capital goods and the composition of the capital goods production. We start with the case in which the two capital goods are perfect substitutes (which happens, for example, when it is costly to change total capital but not each sector's capital stock). The relative price between the two capital goods then is one and the firms in the capital sector are indifferent between different compositions of their productions. The composition of the capital goods production is then entirely determined by the demand for capital goods. Since there are sector-specific externalities, changes in beliefs about future returns can then lead to self-fulfilling changes in the composition of the capital goods production. We continue with the case in which the two capital goods are imperfect substitutes (which happens, for example, when it is costly to change each sector's capital stock). The relative price between the two capital goods then varies with the composition of the capital goods production and the firms in the capital sector are not indifferent between different compositions of their productions. The relative price of one capital good in terms of the other then determines the composition of the capital production. Since that relative price is determined at each point in time by past consumption-savings decisions, changes in beliefs about future returns can no longer lead to self-fulfilling changes in the composition of the capital goods production.

Wen (1998), Guo and Lansing (2002), and Kim (2003) also studied the implications of capital adjustment costs for local indeterminacy, but they employed the one-sector neoclassical growth model with an externality. They all found a threshold result: given a strength of increasing returns that implies local indeterminacy, there is a positive, minimum size of the capital adjustment costs that makes local indeterminacy impossible. ${ }^{2}$ The threshold behavior of the one-sector model is similar to the behavior of the two-sector model when the adjustment costs apply to the total capital stock [Herrendorf and

\footnotetext{
${ }^{2}$ Lahiri (2001) shows that opening up capital mobility has the opposite effect as capital adjustment costs: it decreases the strength of increasing returns required for indeterminacy.
} 
Valentinyi (2003)]. The threshold behavior of the one-sector model is very different from the behavior of the two-sector model when the adjustment costs apply to each sector's capital stock separately and indeterminacy cannot occur for any strength of increasing returns that leaves the aggregate labor demand curve downward sloping. The reason is that adjustment costs of each sector's capital stock lead to imperfect substitutability of the two capital goods, so the results of the present paper apply.

\section{Model Economy}

Consider the following environment. Time is continuous and runs forever. There is a representative households and two representative firms. One representative firm produces a perishable consumption good and the other one produces two new capital goods. The representative household is endowed with the initial capital stocks, with the property rights for the representative firms, and with one unit of time at each instant. We assume that installed capital is sector specific, which is consistent with the evidence collected by Ramey and Shapiro (2001) that it is very costly to reallocate installed capital to other sectors. At each point in time five commodities are traded in sequential markets: the consumption good, the new capital good suitable for the production of consumption goods, the new capital good suitable for the production of new capital goods, working time in the consumption sector, and working time in the capital sector.

The representative household solves:

$$
\begin{aligned}
& \max \max _{t, X_{c t}, X_{x t}, L_{c t}, L_{x t}, K_{c t}, K_{x t} t_{t=0}^{\infty \infty}} \int_{0}^{\infty} e^{-\rho t}\left[\log C_{t}+\left(L-L_{c t}-L_{x t}\right)\right] d t \\
& \text { s.t. } \quad C_{t}+p_{c t} X_{c t}+p_{x t} X_{x t}=\pi_{c t}+\pi_{x t}+w_{c t} L_{c t}+w_{x t} L_{x t}+r_{c t} K_{c t}+r_{x t} K_{x t}, \\
& \\
& \dot{K}_{c t}=X_{c t}-\delta_{c} K_{c t}, \quad \dot{K}_{x t}=X_{x t}-\delta_{x} K_{x t}, \\
& \\
& K_{c 0}=\bar{K}_{c 0} \text { given, } \quad K_{x 0}=\bar{K}_{x 0} \text { given, } \\
& 0 \leq C_{t}, L_{c t}, L_{x t}, X_{c t}, X_{x t}, K_{c t}, K_{x t}, \quad L_{c t}+L_{x t} \leq L .
\end{aligned}
$$


The notation is as follows: $\rho>0$ is the discount rate; $C_{t}$ denotes the consumption good at time $t$ (which is the numeraire); $L \in(0, \infty)$ is the time endowment; the subscripts $c$ and $x$ indicate variables from the consumption and the capital sector; $L_{c t}$ and $L_{x t}$ are the working times, $w_{c t}$ and $w_{x t}$ are the wages, $X_{c t}$ and $X_{x t}$ are the new capital goods, $p_{c t}$ and $p_{x t}$ are the relative prices of the new capital goods, $K_{c t}$ and $K_{x t}$ are the installed capital stocks, $r_{c t}$ and $r_{x t}$ are the real interest rates, $\delta_{c}$ and $\delta_{x}$ are the depreciation rates, and $\pi_{c t}$ and $\pi_{x t}$ are the profits (which will be zero in equilibrium). Two features of the representative household's problem deserve further comment. First, we restrict $X_{c t}$ and $X_{x t}$ to be non-negative, meaning that installed capital is sector specific. Nevertheless the capital stock of a sector can be reduced by not replacing depreciated capital, so close to the steady state (the existence of which we will prove below) the non-negativity constraints will not be binding. Second, we choose the functional form for utility that is most commonly used in the literature. We focus on an infinite equilibrium labor supply elasticity because the existing studies identify this to be the best case for local indeterminacy. An economic justification for an infinite labor supply elasticity is the lottery argument of Hansen (1985) and Rogerson (1988).

Denoting by $\mu_{c t}$ and $\mu_{x t}$ the current value multipliers attached to the accumulation equations (1c), the necessary and sufficient conditions for a solution to the household's problem are (1b)-(1e) and

$$
\begin{aligned}
& \frac{p_{c t}}{C_{t}}=\mu_{c t}, \quad \frac{p_{x t}}{C_{t}}=\mu_{x t}, \\
& C_{t}=w_{c t}=w_{x t}, \\
& \left.\dot{\mu}_{c t} \leq \mu_{c t}\left(\delta_{c}+\rho\right)-\frac{r_{c t}}{C_{t}} \quad \text { (with equality if } X_{c t}>0\right), \\
& \left.\dot{\mu}_{x t} \leq \mu_{x t}\left(\delta_{x}+\rho\right)-\frac{r_{x t}}{C_{t}} \quad \text { (with equality if } X_{x t}>0\right), \\
& \lim _{t \rightarrow \infty} \frac{p_{c t} K_{c t}}{C_{t}} e^{-\rho t}=\lim _{t \rightarrow \infty} \frac{p_{x t} K_{x t}}{C_{t}} e^{-\rho t}=0 .
\end{aligned}
$$

Note that, as usual, the dynamic first-order conditions (2c) and (2d) hold only for $t>0$. Note too that the wage rates will be equalized across sectors but the real interest rates will only be equalized across sectors if the two capital goods are perfect substitutes, in which case their shadow prices are equal. 
We now turn to the production side of the model economy. The problem of the representative firm of the consumption sector is:

$$
\begin{aligned}
\max _{c_{t}, k_{c t}, l_{c t}} & \pi_{c t} \equiv c_{t}-r_{c t} k_{c t}-w_{c t} l_{c t} \\
\text { s.t. } & c_{t}=A_{t} k_{c t}^{a} l_{c t}^{1-a}, \quad c_{t}, l_{c t}, k_{c t} \geq 0,
\end{aligned}
$$

where $A_{t} \geq 0$ denotes total factor productivity in the sector and $a \in(0,1)$. The necessary and sufficient conditions for a solution are (3b) and

$$
\begin{aligned}
r_{c t} & =a A_{t} k_{c t}^{a-1} l_{c t}^{1-a}, \\
w_{c t} & =(1-a) A_{t} k_{c t}^{a} l_{c t}^{-a} .
\end{aligned}
$$

The problem of the representative firm of the capital sector is:

$$
\begin{aligned}
\max _{x_{x t}, x_{c t}, x_{x t}, k_{x t}} & \pi_{x t} \equiv p_{x t} x_{x t}+p_{c t} x_{c t}-r_{x t} k_{x t}-w_{x t} l_{x t} \\
\text { s.t. } & f\left(x_{c t}, x_{x t}\right)=B_{t} k_{x t}^{b} l_{x t}^{1-b}, \quad x_{x t}, x_{c t}, k_{x t}, l_{x t} \geq 0,
\end{aligned}
$$

where $B_{t} \geq 0$ denotes total factor productivity in the sector, $b \in(0,1)$, and $f$ is a twice continuously differentiable function that is non-negative, increasing in both arguments, linear homogeneous, and quasi-convex. ${ }^{3}$ A functional form that satisfies these requirements is

$$
f\left(x_{c t}, x_{x t}\right)=\left(\phi_{c} x_{c t}^{1+\varepsilon}+\phi_{x} x_{x t}^{1+\varepsilon}\right)^{\frac{1}{1+\varepsilon}},
$$

where $\phi_{c}, \phi_{x}$, and $\varepsilon$ are positive constants.

Denoting the multiplier attached to the equation of (5b) by $\lambda_{t}$, the necessary and sufficient conditions

\footnotetext{
${ }^{3}$ Homogeneity is required for the existence of a balanced growth path.
} 
for the solution to problem (5) are (5b) and

$$
\begin{array}{ll}
r_{x t}=\lambda_{t} b B_{t} k_{x t}^{b-1} l_{x t}^{1-b}, & \\
w_{x t}=\lambda_{t}(1-b) B_{t} k_{x t}^{b} l_{x t}^{-b}, & \\
p_{c t} \leq \lambda_{t} \phi_{c}\left(x_{c t}, x_{x t}\right) & \text { (with equality if } x_{c t}>0 \text { ), } \\
p_{x t} \leq \lambda_{t} \phi_{x}\left(x_{c t}, x_{x t}\right) & \text { (with equality if } x_{x t}>0 \text { ), }
\end{array}
$$

where $\phi_{c}$ and $\phi_{x}$ denote the partial derivatives of $f$ with respect to $x_{c t}$ and $x_{x t}$.

The assumption of quasi-convexity implies that for a given $\bar{f} \in \mathbb{R}_{+}$the lower sets $\left\{\left(x_{x t}, x_{c t}\right) \in\right.$ $\left.\mathbb{R}_{+}^{2} \mid f\left(x_{x t}, x_{c t}\right) \leq \bar{f}\right\}$ are convex, so the production possibility frontier between the two new capital goods, $x_{c t}$ and $x_{x t}$, is concave. In other words, the two new capital goods are imperfect substitutes. This is relevant only if the two installed capital goods are also imperfect substitutes, otherwise any reallocation of total capital between the two sectors can be achieved by reallocating installed capital. It is for this reason that we have assumed that installed capital is sector specific. The standard assumption in the literature is that $f$ is linear:

$$
f\left(x_{c t}, x_{x t}\right)=\phi_{c} x_{c t}+\phi_{x} x_{x t},
$$

where $\phi_{c}$ and $\phi_{x}$ are positive constants, which are often set to one. ${ }^{4}$ If $f$ is linear, then the production possibility frontier between the two new capital goods is linear too. In other words, the two new capital goods are perfect substitutes. If this is the case, then it is irrelevant for the local stability properties whether the two installed capital goods are perfect or imperfect substitutes. The reason is that installed capital depreciates, so close to the steady state any change in the capital stocks of the two sectors can be achieved by a corresponding change in the composition of the new capital production. In any case, we find it convenient to maintain sector-specificity also when we study perfect substitutability of new capital goods. ${ }^{5}$

\footnotetext{
${ }^{4}$ The choice of $\phi_{c}$ and $\phi_{x}$ amounts to a choice of the units in which $x_{c t}$ and $x_{x t}$ are denominated. This choice does not matter for the local stability properties of the steady state.

${ }^{5}$ Note that at $t=0$ both $k_{c 0}$ and $k_{x 0}$ are given because installed capital is assumed to be sector-specific. However, this does not invalidate the previous argument.
} 
The total factor productivities are specified so that there can be positive externalities at the level of each sector:

$$
A_{t}=k_{c t}^{\theta_{c} a} l_{c t}^{\theta_{c}(1-a)}, \quad B_{t}=k_{x t}^{\theta_{x} b} l_{x t}^{\theta_{x}(1-b)}
$$

where $\theta_{c}, \theta_{x} \geq 0$. Substituting (9) back into the production functions, the sectors' aggregate outputs become:

$$
\begin{array}{lll}
c_{t}=k_{c t}^{\alpha_{1}} l_{x t}^{\alpha_{2}}, & \alpha_{1} \equiv\left(1+\theta_{c}\right) a, & \alpha_{2} \equiv\left(1+\theta_{c}\right)(1-a), \\
x_{t}=k_{x t}^{\beta_{1}} l_{x t}^{\beta_{2}}, & \beta_{1} \equiv\left(1+\theta_{x}\right) b, & \beta_{2} \equiv\left(1+\theta_{x}\right)(1-b) .
\end{array}
$$

Note that (9) implies that the externalities on capital and labor are the same. ${ }^{6}$ Note too that the externalities are not taken into account by the firms, so a competitive equilibrium exists. In equilibrium, profits are zero and the capital and labor shares are the usual ones: $\frac{r_{c t} k_{x t}}{c_{t}}=a, \frac{w_{c} l_{x c}}{c_{t}}=1-a, \frac{r_{x t} k_{x t}}{k_{t}}=b$, $\frac{w_{x t} l_{x t}}{k_{t}}=1-b$. Moreover, in equilibrium, the total factor productivities on which the firms base their decisions must be equal to those that results from these decisions:

Definition 1 (Competitive equilibrium) A competitive equilibrium is prices $\left\{w_{c t}, w_{x t}, r_{c t}, r_{x t}, p_{c t}, p_{x t}\right\}_{t=0}^{\infty}$, an allocation $\left\{C_{t}, c_{t}, X_{c t}, x_{c t}, X_{x t}, x_{x t}, L_{c t}, l_{c t}, L_{x t}, l_{x t}, K_{c t}, k_{c t}, K_{x t}, k_{x t}\right\}_{t=0}^{\infty}$, and total factor productivities $\left\{A_{t}\right.$, $\left.B_{t}\right\}_{t=0}^{\infty}$ such that:

(i) $\left\{C_{t}, X_{c t}, X_{x t}, L_{c t}, L_{x t}, K_{c t}, K_{x t}\right\}_{t=0}^{\infty}$ solve the problem of the representative household, (1), that is, (2a)(2e) hold;

(ii) $\left\{c_{t}, l_{c t}, k_{c t}\right\}_{t=0}^{\infty}$ solve the problem of the representative firm of the consumption sector, (3), that is, (4a)-(4b) hold;

(iii) $\left\{x_{x t}, x_{c t}, l_{x t}, k_{x t}\right\}_{t=0}^{\infty}$ solve the problem of the representative firm of the capital sector, (5), that is, (7a)-(7d) hold;

(iv) markets clear, that is, $C_{t}=c_{t}, X_{c t}=x_{c t}, X_{x t}=x_{x t}, L_{c t}=l_{c t}, L_{x t}=l_{x t}, K_{c t}=k_{c t}, K_{x t}=k_{x t}$;

(v) $A_{t}$ and $B_{t}$ are determined consistently, that is, the two equations in (9) hold.

\footnotetext{
${ }^{6}$ The results of Harrison and Weder (2001) suggest that imposing this constraint does not affect in an important way the stability properties of the steady state of the two-sector neoclassical growth model without capital adjustment costs.
} 


\section{Analytical Results}

\subsection{Local stability properties}

We start by establishing that there is a unique steady state and by deriving the reduced-form equilibrium dynamics nearby.

\section{Proposition 1 (Reduced-form dynamics)}

(i) There is a unique steady state.

(ii) If $f$ is linear, then there is a neighborhood of the steady state such that the equilibrium reducedform dynamics can be described by the dynamics of the state variable $k_{t} \equiv \phi_{c} k_{c t}+\phi_{x} k_{x t}$ and the dynamics of the control variable $\mu_{c t}$.

(iii) If $f$ is strictly quasi convex, then there is a neighborhood of the steady state such that the equilibrium reduced-form dynamics can be described by the dynamics of the two state variables $k_{c t}$ and $k_{x t}$ and the two control variables $\mu_{c t}$ and $\mu_{x t}$.

Proof. See the Appendix A.

The proposition shows that the equilibrium reduced-form dynamics close to the steady state are two dimensional when $f$ is linear and four dimensional when $f$ is strictly quasi convex. The reasons are as follows. With a linear $f$ the two capital goods are perfect substitutes, so only the total capital stock and its shadow price are needed to describe the dynamics. With a strictly quasi-convex $f$, the two capital goods are imperfect substitutes, so both of them and two shadow prices are needed to describe the dynamics.

We now explore analytically the stability properties of the steady state. The steady state is saddlepath stable if there are as many stable roots (i.e. roots with negative real part) as states and as many unstable roots (i.e. roots with positive real part) as controls. It is stable if there are more stable roots than states and it is unstable if there more unstable roots than controls. If the steady state is saddle-path stable then the equilibrium is determinate, that is, given the initial capital stocks close to the steady 
state values there are unique initial shadow prices such that the model economy converges to the steady state. If the steady state is stable, then the equilibrium is locally indeterminate, that is, given the initial capital stocks close to the steady state values there exists a continuum of shadow prices such that the model economy converges to the steady state. Since it is not feasible to compute analytically the four eigenvalues, we will only compute the determinant and the trace of the linearization of the reducedform equilibrium dynamics at the steady state. Although this does not allow for a full characterization of the local stability properties, it provides important information because the determinant equals the product of the eigenvalues and the trace equals the sum of the real parts of the eigenvalues (complex eigenvalues occur in conjugates, implying that the imaginary parts cancel in the summation). This leads to the next proposition, which constitutes the main result of our paper.

Proposition 2 (Local stability properties of the steady state) Suppose that $b<1-b$ and $\theta_{x} \in\left[0, \frac{1-b}{b}\right.$ ).

(i) Suppose that $f$ is linear.

There are constants $\underline{\theta}_{x} \in\left(0, \frac{b}{1-b}\right)$ and $\bar{\theta}_{x} \in\left(-\frac{\rho b}{\rho b+\delta_{x}}, \frac{1-b}{b}\right)$ such that:

(i.a) if

$$
\left(\rho+\delta_{x}\right)\left[\rho+(1-b) \delta_{x}\right]>\frac{\rho b+\delta_{x}}{\rho} b \delta_{c}\left(\rho+\delta_{c}\right)
$$

then $\underline{\theta}_{x}<\bar{\theta}_{x}$ and the steady state is saddle-path stable for $\theta_{x} \in\left[0, \underline{\theta}_{x}\right)$, stable for $\theta_{x} \in\left(\underline{\theta}_{x}, \bar{\theta}_{x}\right)$, and unstable for $\theta_{x} \in\left(\bar{\theta}_{x}, \frac{1-b}{b}\right)$;

(i.b) if

$$
\left(\rho+\delta_{x}\right)\left[\rho+(1-b) \delta_{x}\right]<\frac{\rho b+\delta_{x}}{\rho} b \delta_{c}\left(\rho+\delta_{c}\right)
$$

then $\bar{\theta}_{x}<0<\underline{\theta}_{x}$ and the steady state is saddle-path stable for $\theta_{x} \in\left[0, \underline{\theta}_{x}\right)$ and stable for $\theta_{x} \in\left(\underline{\theta}_{x}, \frac{1-b}{b}\right)$.

(ii) Suppose that $f$ is strictly quasi-convex.

(ii.a) $\theta_{x} \in\left[0, \frac{b}{1-b}\right)$ is a necessary condition for the steady state to be saddle-path stable;

(ii.b) $\theta_{x} \in\left(\frac{b}{1-b}, \frac{1-b}{b}\right)$ is a necessary condition for the steady state to be stable. 
Proof. See the Appendix B.

We begin the discussion by noting that calibrations of our two-sector model that are typical in the literature are consistent with the assumptions $b<1-b$ and $\theta_{x}<\frac{1-b}{b}$. ${ }^{7}$ The inequality $b<1-b$ ensures that the capital share in the capital sector's income is smaller than one half and that $\frac{b}{1-b}<\frac{1-b}{b}$. The inequality $\theta_{x}<\frac{1-b}{b}$ ensures that the aggregate returns to capital are not too large so there cannot be endogenous growth in steady state. As pointed out before, there are two relevant subcases of $\theta_{x}<\frac{1-b}{b}$ : for $\theta_{x} \in\left[0, \frac{b}{1-b}\right)$ the aggregate labor demand curve of the capital sector slopes downward and for $\theta_{x} \in\left(\frac{b}{1-b}, \frac{1-b}{b}\right)$ it slopes upward.

We continue the discussion of this proposition with part (i), the case of a linear $f$. It says that if the aggregate labor demand curve in the capital sector slopes downward, then the steady state can be saddle-path stable, stable, or unstable. ${ }^{8}$ The key part of this statement is that a linear $f$ allows for a stable steady state and therefore for local indeterminacy at the steady state when the aggregate labor demand curve in the capital sector slopes downward and capital is sector specific capital. ${ }^{9}$ This replicates the result of the recent literature on self-fulfilling business cycles; see for example Benhabib and Farmer (1996) and Harrison and Weder (2001).

We conclude the discussion with part (ii), the case of a strictly-quasi convex $f$. It says that if the aggregate labor demand curve in the capital sector slopes upward, then the steady state can be stable or unstable but not saddle-path stable; if the aggregate labor demand curve in the capital sector slopes downward, then the steady state can be saddle-path stable or unstable but not stable. Thus, a strictly-quasi convex $f$ rules out local indeterminacy at the steady state if the aggregate labor demand curve slopes downward. This is our key analytical result, which holds for any strictly quasi-convex $f$. In other words, the local stability properties of the two-sector neoclassical growth model with strictly quasi-convex $f$ differ strikingly from those with a linear $f$. In fact, the local stability proper-

\footnotetext{
${ }^{7}$ Below we will discuss calibration issues in more detail.

${ }^{8}$ Using the results from Appendix B, it is easy to verify that if $\rho\left[\rho+(1-b) \delta_{x}\right]>b \delta_{c}\left(\rho+\delta_{c}\right)$, then $\bar{\theta}<\frac{b}{1-b}$ and the steady state can be unstable under downward sloping aggregate labor demand curve.

${ }^{9}$ It has already been shown by Christiano (1995) for a discrete time version of a two-sector real business cycle model that the stability properties of the steady state do not depend on whether capital is sector specific or fully mobile across sectors.
} 
ties of the two-sector model with strictly quasi-convex $f$ are much more like those of the one-sector neoclassical growth model without capital adjustment costs, in which local indeterminacy requires an upward-sloping aggregate labor demand curve [Benhabib and Farmer (1994)].

\subsection{Intuition}

Here we seek to understand why imperfect substitutability precludes the possibility of local indeterminacy when the aggregate labor demand of the capital sector slopes downward. We start by demonstrating that as the model economies with strictly quasi-convex $f$ converge to that with a linear $f$, the steady states behave continuously. So a discontinuity at the steady state cannot be the explanation for our results. In order to be able to establish this, we need to specify what we mean by convergence.

Definition 2 (Convergence to a linear $f$ ) Consider a linear function $f: \mathbb{R}_{+}^{2} \longrightarrow \mathbb{R}_{+}$with $f\left(x_{c t}, x_{x t}\right)$, $=f_{c} x_{c t}+f_{x} x_{x t}$ where $f_{c}, f_{x} \geq 0$, denote the steady state values of the new capital goods in the associated model economy by $\left(x_{c}, x_{x}\right)$, and let $U\left(x_{c}, x_{x}\right)$ be an open neighborhood of $\left(x_{c}, x_{x}\right)$. Furthermore, consider a sequence $\left\{f_{i}\right\}_{i=1}^{\infty}$ of functions $f_{i}: \mathbb{R}_{+}^{2} \longrightarrow \mathbb{R}_{+}$that are non-negative, linear homogeneous, twice continuously differentiable, and strictly quasi-convex.

We say that $\left\{f_{i}\right\}_{i=1}^{\infty}$ converges to $f$ on $U\left(x_{c}, x_{x}\right)$ if and only if each of $\left\{f_{i}\right\}_{i=1}^{\infty},\left\{f_{c, i}\right\}_{i=1}^{\infty},\left\{f_{x, i}\right\}_{i=1}^{\infty},\left\{f_{c c, i}\right\}_{i=1}^{\infty}$, $\left\{f_{x x, i}\right\}_{i=1}^{\infty}$, and $\left\{f_{c x, i}\right\}_{i=1}^{\infty}$ converge in the supremum norm defined over $U\left(x_{c}, x_{x}\right)$ to $f, f_{c}, f_{x}, f_{c c}, f_{x x}$, and $f_{c x}$, respectively.

Proposition 3 (Continuity of the steady states) Consider a sequence of functions $\left\{f_{i}\right\}_{i=1}^{\infty}$ of the form described in Definition 2. Then the sequence of the steady states of the model economies with $f_{i}$ converges to the steady state of the model economy with $f$.

Proof. See the Appendix C.

To find the explanation for our results, it is useful to recall how with perfect substitutability local indeterminacy can occur for mild strengths of the externality. ${ }^{10}$ To this end, suppose the model economy

\footnotetext{
${ }^{10}$ The arguments of this section follow Christiano (1995).
} 
is on an equilibrium path to the steady state and ask whether there can be another equilibrium path with the same initial capital stock but temporarily higher capital stocks subsequently. Such a path requires a different composition of capital output: initially more capital goods for the capital sector and then fewer capital goods for the capital sector. This results in initially lower and then higher capital stocks in the consumption sector. Consumption growth is therefore first lower and then higher, which is optimal for the household only if the returns on installed capital are initially higher and then lower. Since with perfect substitutability the relative price of the two new capital goods is constant irrespective of the composition of capital output, only the shape of the aggregate production possibility frontier (ppf henceforth) between consumption and the composite capital good matters. The aggregate ppf is strictly convex, so a lower ratio of consumption to composite capital good is associated with a lower relative price of the composite capital good in terms of consumption and vice versa. Along the alternative path, capital gains can therefore generate the required movements of the returns to capital.

Two crucial ingredients bring about the capital gains when the two capital goods are perfect substitutes. First, the aggregate ppf between consumption and the composite capital good is strictly convex at the steady state. This ingredient is also present when the two capital goods are imperfect, but arbitrarily close, substitutes.

Proposition 4 (Continuity of the PPF) Consider a sequence of functions $\left\{f_{i}\right\}_{i=1}^{\infty}$ of the form described in Definition 2. Providing $x_{c t}, x_{x t}>0$, the sequence of the production possibility frontiers of the economies with $f_{i}$ converges on $U\left(x_{c}, x_{x}\right)$ to the production possibility frontier of the model economy with $f$.

\section{Proof. See the Appendix D.}

The second crucial ingredient that brings about the capital gains with perfect substitutability is the indeterminate composition of the capital goods production. Formally this follows from the fact that the relative price of the two capital goods is constant and so the firm is indifferent between different compositions of its capital production. Hence, the household's demand determines the composition of the capital production, implying that changes in beliefs about future returns can be accommodated by 
changes in the composition of the capital goods production. This second ingredient is not present when the two capital goods are imperfect substitutes. The reason is that the relative price of one new capital good in terms of the other then determines the composition of the capital goods production. Formally, this follows by combining (2a), (7c), and (7d) (the last two with equality):

$$
\frac{p_{c t}}{p_{x t}}=\frac{\mu_{c t}}{\mu_{x t}}=\frac{f_{c}\left(\frac{x_{c t}}{x_{x t}}, 1\right)}{f_{x}\left(\frac{x_{c t}}{x_{x t}}, 1\right)} .
$$

To see how this rules out local indeterminacy, assume that $\delta_{c}=\delta_{x}$, for simplicity, and use the arbitrage conditions (2c) and (2d) together with (2a) to derive:

$$
\frac{r_{c t}}{p_{c t}}+\frac{\dot{p}_{c t}}{p_{c t}}=\frac{r_{x t}}{p_{x t}}+\frac{\dot{p}_{x t}}{p_{x t}}
$$

Consequently,

$$
\frac{d}{d t}\left(\frac{p_{c t}}{p_{x t}}\right)=\frac{p_{c t}}{p_{x t}}\left(\frac{r_{x t}}{p_{x t}}-\frac{r_{c t}}{p_{c t}}\right)
$$

Consider now an equilibrium path that converges to the steady state and ask whether there can be another equilibrium path that too converges to the steady state and that initially has less capital allocated to the consumption sector, so initially $x_{c t} / x_{x t}$ is smaller. (11) shows that initially $p_{c t} / p_{x t}$ must then be smaller too. Since with less capital in the consumption sector the marginal product of capital in the consumption sector is larger than in the capital sector, we also have that initially

$$
\frac{r_{x t}}{p_{x t}}-\frac{r_{c t}}{p_{c t}}<0
$$

The arbitrage equation (12) therefore implies that $p_{c t} / p_{x t}$ must decrease further and (11) implies that $x_{c t} / x_{x t}$ must decrease further, and so on and so forth. Consequently, along the alternative path, the capital stock of the consumption sector is ever decreasing, implying that the alternative path cannot converge to the steady state and thus violates the transversality condition. Therefore, it cannot be an equilibrium path. 
It is important to emphasize that capital gains are possible irrespective of whether we have perfect or imperfect substitutability. The difference is that with perfect substitutability the capital gains have no direct effect on the composition of the capital goods production. In contrast, with imperfect substitutability the capital gains affect the composition of the capital goods production. This is the key feature of our model that rules out local indeterminacy for downward-sloping aggregate labor demand curve.

\section{Numerical Results}

The analytical results derived so far for imperfect substitutability show that a necessary condition for local indeterminacy is an upward-sloping aggregate labor demand curve and a necessary condition for determinacy is a downward-sloping aggregate labor demand curve. Since our dynamical system is four dimensional, it is impossible to fully characterize the local stability properties at the steady state analytically. Thus, we now calibrate the model and then compute the four eigenvalues numerically. We use the functional forms and most parameter values of Huffman and Wynne (1999), who calibrate a two-sector model similar to our's but with constant returns in both sectors, $\theta_{c}=\theta_{x}=0$. This difference does not affect the usefulness of their calibration for our purposes because the degrees of increasing returns do not affect the calibration of the other parameters. The specific assumptions of Huffman and Wynne is that $f$ is of the form displayed in (6). Using quarterly, postwar, one-digit US data, Huffman and Wynne calibrate $\delta_{c}=0.018, \delta_{x}=0.020, a=0.41, b=0.34$, and $\rho=0.01$. Moreover, they calibrate $\varepsilon=0.1$ or $\varepsilon=0.3$, depending on the procedure. We adopt their calibration except we set $\rho=0.02$. The reason is that $\rho=0.02$ implies more reasonable steady-state ratios between consumption and output and between capital and output than $\rho=0.01: 0.81$ and 2.59 versus 0.75 and $3.45 .^{11}$

The equations for the linearization with imperfect substitutability, (B.7)-(B.9), show that the re-

\footnotetext{
${ }^{11}$ To avoid confusion, note that the imperfect substitutability of the two capital goods can come from intratemporal capital adjustment costs, which make changes in the allocations of capital across sectors costly but leave changes in the total capital stock costless. Huffman and Wynne (1999) adopt this interpretation.
} 
Figure 1: Local stability if capital goods are imperfect substitutes and $\rho=0.02, \delta_{c}=0.018, \delta_{x}=0.020, a=0.41, b=0.34$.

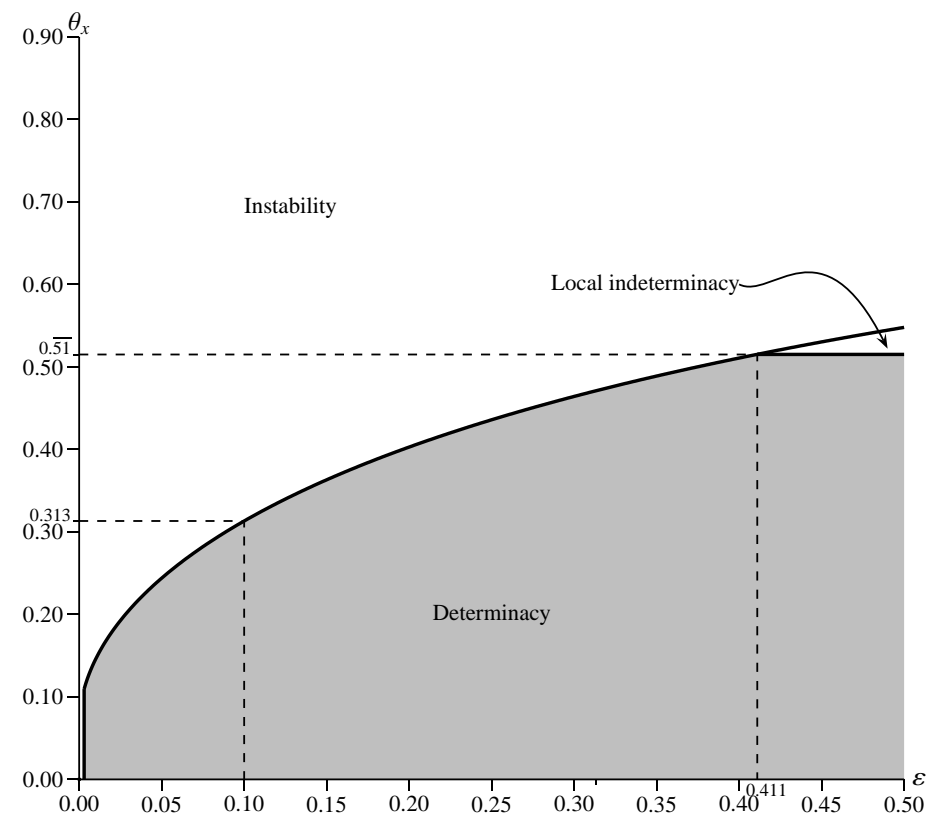

duced-form equilibrium dynamics are independent of $\theta_{c}$, so we do not need to choose a value for $\theta_{c}{ }^{12}$ The available evidence on $\theta_{x}$ is somewhat mixed. However, it is clear that Hall's (1988) initial estimates of aggregate increasing returns of about 0.5 were upward biased. More recent empirical studies find estimates between constant returns and milder increasing returns up to 0.3; see e.g. Bartelsman et al. (1994), Burnside et al. (1995), Burnside (1996), Basu and Fernald (1997), and Harrison (2003). According to Basu and Fernald (1997) and Harrison (2003) these aggregate increasing returns are mainly due to increasing returns in the capital sector; specifically they estimate non-durable manufacturing to have constant (or decreasing) returns and durable manufacturing to have mildly increasing returns. Since $\theta_{x}$ is a key parameter determining the local stability properties of the steady state and since it is hard to draw a sharp line between empirically plausible and implausible values for it, we will vary it extensively together with the other key parameter $\varepsilon$. Specifically, we will explore the local stability properties of the steady state for all $\theta_{x} \in(0.000,0.900)$ and $\varepsilon \in(0.000,0.500) .^{13}$

\footnotetext{
${ }^{12}$ This is standard, see Weder (2000) and Harrison (2001).

${ }^{13} \varepsilon=0.000000001$ is the closest value to zero that we use in these computations.
} 
Our numerical results are reported in Figure 1. The figure displays the stability properties of the economy for $\varepsilon>0$ only, which is indicated by the small distance between the shaded area and the vertical axis. The numerical results confirm the analytical one that an upward-sloping (downward-sloping) aggregate labor demand curve in the capital sector is a necessary condition for local indeterminacy (determinacy). ${ }^{14}$ The numerical results go beyond the analytical ones in three respects. First, they show that an upward-sloping (downward-sloping) aggregate labor demand curve in the capital sector becomes a sufficient condition for local indeterminacy (determinacy) when the substitutability between the capital goods are sufficiently low ( $\varepsilon \geq 0.411$ ). Second, they show that for capital adjustment costs within the range calibrated by Huffman and Wynne, $\varepsilon \in[0.1,0.3]$, the steady state is determinate if the increasing returns do not exceed 0.313 . The range $\theta_{x} \in[0,0.313]$ includes most values of increasing returns that are usually considered reasonable. So, given $\varepsilon \in[0.1,0.3]$, the local stability properties with a strictly quasi-convex $f$ are summarized by determinacy for every empirically plausible specification of $\theta_{x}$. Third, our numerical results show that $\varepsilon=0.000000001$ makes the equilibrium determinate for $\theta_{x} \in(0,0.078)$. In contrast, Proposition 2 shows that for $\varepsilon=0$ the equilibrium is locally indeterminate for $\theta_{x} \in(0.053,0.078)$. Thus, the steady state with small degree of imperfect substitutability is saddlepath stable in the region of increasing returns in which the steady state with perfect substitutability is stable. $^{15}$

We complete this section with a brief discussion of the robustness of our numerical findings, which we have explored in two directions. First, we have shown that our numerical determinacy result survives for reasonable variations of the parameter values used above. Second, we have shown that our numerical determinacy result survives when the imperfect substitutability comes from capital adjustment costs of the form suggested by Lucas and Prescott (1971). The details can be found in Herrendorf and Valentinyi (2003).

\footnotetext{
${ }^{14}$ For the calibration used here the equilibrium labor demand curve slopes upward if and only if $\theta_{x}>0 . \overline{51}$.

${ }^{15}$ Note the possibility for global indeterminacy, which we do not pursue any further in this paper. This follows from the additional piece of information that at the bifurcation to "instability" two of the eigenvalues are complex and their real parts change sign, that is, a Hopf bifurcation occurs. The Hopf bifurcation theorem implies the existence of limit cycles, which may or may not be stable. If they are stable, then a form of global indeterminacy occurs.
} 


\section{Conclusion}

We have explored the conditions under which indeterminacy of equilibrium occurs near the steady state in a class of two-sector neoclassical growth models with sector-specific externalities. Our main finding has been that imperfect substitutability of the two new capital goods precludes local indeterminacy for every empirically plausible specification of the model parameters. This analytical result contrasts sharply with the standard result that with perfect substitutability local indeterminacy can occur in the two-sector model for a wide range of plausible parameter values. It can be interpreted to mean that local indeterminacy is not a robust property of the class of two-sector neoclassical growth models with sector-specific externalities. We conjecture that this result is likely to carry over to models with more than two sectors and more than two capital goods.

Our findings are relevant for several reasons. To begin with, if local indeterminacy is impossible for plausible specifications of the parameter values, then self-fulfilling business cycles are impossible for plausible specifications of the parameter values. This has important implications for the debate about whether or not government policy should aim to stabilize business cycles; see Christiano and Harrison (1999). Second, models from the class of two-sector neoclassical growth models that we have studied here are widely used; see for example Fisher (1997), Huffman and Wynne (1999), and Boldrin et al. (2001). Our results provide a better understanding of the local stability properties of this important class of models. Finally, the results of this paper contribute to a recent debate about the robustness of multiple and indeterminate equilibria. Even though Morris and Shin (1998) and Herrendorf et al. (2000) studied rather different environments with externalities, they share a common theme with the present paper: the introduction of frictions can substantially reduce the scope for the multiplicity or local indeterminacy of equilibrium. 


\section{Appendix}

\section{A Proof of Proposition 1}

\section{A.1 Strictly quasi-convex $f$}

\section{A.1.1 Reduced-form dynamics}

Suppose that all first-order conditions hold with equality. (1c) and (2b)-(2d) then imply

$$
\begin{array}{ll}
\dot{k}_{c t}=x_{c t}-\delta_{c} k_{c t}, & \dot{k}_{x t}=x_{x t}-\delta_{x} k_{x t}, \\
\dot{\mu}_{c t}=\mu_{c t}\left(\delta_{c}+\rho\right)-\frac{r_{c t}}{w_{c t}}, & \dot{\mu}_{x t}=\mu_{x t}\left(\delta_{x}+\rho\right)-\frac{r_{x t}}{w_{x t}} .
\end{array}
$$

To represent the model economy as a dynamical system in $k_{c t}, k_{x t}, \mu_{c t}$, and $\mu_{x t}$, we need to express all endogenous variables, i.e. $x_{c t}, x_{x t}, l_{c t}, l_{x t}, r_{c t}, r_{x t}, p_{c t}, p_{x t}, w_{c t}$, and $w_{x t}$, as functions of these four variables. Establishing this is the first step of the proof.

To begin with, note that (2a) implies that $\frac{p_{c t}}{p_{x t}}=\frac{\mu_{c t}}{\mu_{x t}}$, so (7c) and (7d) (with equality) together with the strict quasi-convexity of $t$ imply that there is a function $g$ such that:

$$
g\left(\frac{\mu_{c t}}{\mu_{x t}}\right) \equiv\left(\frac{f_{c}}{f_{x}}\right)^{-1}\left(\frac{\mu_{c t}}{\mu_{x t}}\right)=\frac{x_{c t}}{x_{x t}} .
$$

Next, observe that dividing (4a) by (4b) and (7a) by (7b) and using (A.3a), we can express the factor price ratios as functions of the corresponding factors:

$$
\frac{r_{c t}}{w_{c t}}=\frac{a}{1-a} \frac{l_{c t}}{k_{c t}} \quad \frac{r_{x t}}{w_{x t}}=\frac{b}{1-b} \frac{l_{x t}}{k_{x t}} .
$$

Now, we derive labor in the consumption sector. Combining (2b), the first equation of $(3 b)$, and (4b) gives:

$$
l_{c t}=1-a
$$


Turning to labor in the capital sector, observe that (2a) and (2b) imply $1=\mu_{x t} \frac{w_{x t}}{p_{x t}}$. Substituting (7b) and (7b) into this leads to

$$
1=(1-b) \mu_{x t} k_{x t}^{\beta_{1}} l_{x t}^{\beta_{2}-1}\left[f_{x}\left(g\left(\frac{\mu_{c t}}{\mu_{x t}}\right), 1\right)\right]^{-1}
$$

where we used the fact that $f(\cdot, \cdot)$ is homogeneous of degree one, and (A.2a). Rearranging leads to the reduced form for labor in the capital sector:

$$
l_{x t}=l_{x}\left(k_{x t}, \mu_{c t}, \mu_{x t}\right) \equiv\left[(1-b) \mu_{x t}\right]^{\frac{1}{1-\beta_{2}}} f_{x}\left(g\left(\frac{\mu_{c t}}{\mu_{x t}}\right), 1\right)^{\frac{1}{\beta_{2}-1}} k_{x t}^{\frac{\beta_{1}}{1-\beta_{2}}}
$$

Substituting (A.3a) and (A.3b) into (A.2b) for $l_{c}$ and $l_{x}$, rearranging and plugging the result into (A.1b) gives:

$$
\begin{aligned}
& \dot{\mu}_{c t}=F_{\mu c}\left(k_{c t}, k_{x t}, \mu_{c t}, \mu_{x t}\right) \equiv\left(\rho+\delta_{c}\right) \mu_{c t}-\frac{a}{k_{c t}}, \\
& \dot{\mu}_{x t}=F_{\mu x}\left(k_{c t}, k_{x t}, \mu_{c t}, \mu_{x t}\right) \equiv\left(\rho+\delta_{x}\right) \mu_{x t}-\frac{b}{1-b}\left[(1-b) \mu_{x t}\right]^{\frac{1}{1-\beta_{2}}} f_{x}\left(g\left(\frac{\mu_{c t}}{\mu_{x t}}\right), 1\right)^{\frac{1}{\beta_{2}-1}} k_{x t}^{\frac{\beta_{1}+\beta_{2}-1}{1-\beta_{2}}} .
\end{aligned}
$$

Next, we derive the expressions for each type of investment. Substituting (9) and (A.2a) into the equation of $(5 b)$ gives

$$
k_{x t}^{\beta_{1}} l_{x t}^{\beta_{2}}=x_{c t} \frac{f\left(g\left(\frac{\mu_{c t}}{\mu_{x t}}\right), 1\right)}{g\left(\frac{\mu_{c t}}{\mu_{x t}}\right)}=x_{x t} f\left(g\left(\frac{\mu_{c t}}{\mu_{x t}}\right), 1\right) .
$$

To eliminate $l_{x t}$ from these expressions, we use (A.3b). Solving afterwards for $x_{c t}$ and $x_{x t}$ gives:

$$
\begin{aligned}
& x_{c t}=x_{c}\left(k_{x t}, \mu_{c t}, \mu_{x t}\right) \equiv\left[(1-b) \mu_{x t}\right]^{\frac{\beta_{2}}{1-\beta_{2}}} \frac{g\left(\frac{\mu_{c t}}{\mu_{x t}}\right) f_{x}\left(g\left(\frac{\mu_{c t}}{\mu_{x t}}\right), 1\right)^{\frac{\beta_{2}}{\beta_{2}-1}}}{f\left(g\left(\frac{\mu_{c t}}{\mu_{x t}}\right), 1\right)} k_{x t}^{\frac{\beta_{1}}{1-\beta_{2}}}, \\
& x_{x t}=x_{x}\left(k_{x t}, \mu_{c t}, \mu_{x t}\right) \equiv\left[(1-b) \mu_{x t}\right]^{\frac{\beta_{2}}{1-\beta_{2}}} \frac{f_{x}\left(g\left(\frac{\mu_{c t}}{\mu_{x t}}\right), 1\right)^{\frac{\beta_{2}}{\beta_{2}-1}}}{f\left(g\left(\frac{\mu_{c t}}{\mu_{x t}}\right), 1\right)} k_{x t}^{\frac{\beta_{1}}{1-\beta_{2}}} .
\end{aligned}
$$

Substituting the above reduced forms for $x_{c t}, x_{x t}$, into (A.1a) and rearranging, we find the reduced-form 
equilibrium dynamics:

$$
\begin{aligned}
& \dot{k}_{c t}=F_{k c}\left(k_{c t}, k_{x t}, \mu_{c t}, \mu_{x t}\right) \equiv\left[(1-b) \mu_{x t}\right]^{\frac{\beta_{2}}{1-\beta_{2}}} \frac{g\left(\frac{\mu_{c t}}{\mu_{x t}}\right) f_{x}\left(g\left(\frac{\mu_{c t}}{\mu_{x t}}\right), 1\right)^{\frac{\beta_{2}}{\beta_{2}-1}}}{f\left(g\left(\frac{\mu_{c t}}{\mu_{x t}}\right), 1\right)} k_{x t}^{\frac{\beta_{1}}{1-\beta_{2}}}-\delta_{c} k_{c t}, \\
& \dot{k}_{x t}=F_{k x}\left(k_{c t}, k_{x t}, \mu_{c t}, \mu_{x t}\right) \equiv\left[(1-b) \mu_{x t}\right]^{\frac{\beta_{2}}{1-\beta_{2}}} \frac{f_{x}\left(g\left(\frac{\mu_{c t}}{\mu_{x t}}\right), 1\right)^{\frac{\beta_{2}}{\beta_{2}-1}}}{f\left(g\left(\frac{\mu_{c t}}{\mu_{x t}}\right), 1\right)} k_{x t}^{\frac{\beta_{1}}{1-\beta_{2}}}-\delta_{x} k_{x t} .
\end{aligned}
$$

\section{A.1.2 Existence and uniqueness of steady state}

Representing variables in steady state by dropping the time index $t$ and assuming that all first-order conditions hold with equality, the steady state versions of (A.4b) and (A.4d) are found to be:

$$
\begin{gathered}
\delta_{x} k_{x}^{\frac{1-\beta_{1}-\beta_{2}}{1-\beta_{2}}}=\left[(1-b) \mu_{x}\right]^{\frac{\beta_{2}}{1-\beta_{2}}} \frac{f_{x}\left(g\left(\frac{\mu_{c}}{\mu_{x}}\right), 1\right)^{\frac{\beta_{2}}{\beta_{2}-1}}}{f\left(g\left(\frac{\mu_{c}}{\mu_{x}}\right), 1\right)}, \\
\left(\rho+\delta_{x}\right) k_{x}^{\frac{1-\beta_{1}-\beta_{2}}{1-\beta_{2}}}=b\left[(1-b) \mu_{x}\right]^{\frac{\beta_{2}}{1-\beta_{2}}} f_{x}\left(g\left(\frac{\mu_{c}}{\mu_{x}}\right), 1\right)^{\frac{1}{\beta_{2}-1}} .
\end{gathered}
$$

Dividing the second equation by the first one leads to

$$
\frac{\rho+\delta_{x}}{b \delta_{x}}=\frac{f\left(g\left(\frac{\mu_{c}}{\mu_{x}}\right), 1\right)}{f_{x}\left(g\left(\frac{\mu_{c}}{\mu_{x}}\right), 1\right)} .
$$

Given the assumed properties of $f$, this expression can be solved uniquely for $\frac{\mu_{c}}{\mu_{x}}$, so the steady state shadow price ratio is uniquely determined by the parameters of the model. ¿From now on we will therefore write $f, f_{x}$, and $g$ for the unique steady state values of these functions. We can then write (A.4a), (A.4c), and (A.4d) evaluated at the steady state as follows:

$$
\begin{aligned}
\mu_{c t} & =\frac{a}{\rho+\delta_{c}} k_{c t}^{-1}, \\
\delta_{c} k_{c} & =\frac{\left[(1-b) \mu_{x}\right]^{\frac{\beta_{2}}{1-\beta_{2}}}}{f} \frac{\beta_{2}}{f_{x}-1} k_{x}^{\frac{\beta_{1}}{1-\beta_{2}}}, \\
\delta_{x} k_{x} & =\frac{\left[(1-b) \mu_{x}\right]^{\frac{\beta_{2}}{1-\beta_{2}}} f_{x}^{\frac{\beta_{2}}{\beta_{2}-1}}}{f} k_{x}^{\frac{\beta_{1}}{1-\beta_{2}}} .
\end{aligned}
$$


To show uniqueness, we will show that $k_{c}, \mu_{x}$, and $\mu_{c}$ are functions of $k_{x}$. We will then show that $k_{x}$ is uniquely determined by the parameters of the model. Dividing (A.7b) by (A.7c) gives $k_{c}$ as a function of $k_{x}$ :

$$
k_{c}=\frac{\delta_{x}}{\delta_{c} g} k_{x} .
$$

Since from (A.7a) $\mu_{c}$ is a function of $k_{c}$, (A.8) implies that $\mu_{c}$ is a function of $k_{x}$. Since from (A.6) $\mu_{x}$ is a function of $\mu_{c},(\mathrm{~A} .8)$ implies that $\mu_{x}$ is a function of $k_{x}$. Finally, substituting $\mu_{x}\left(k_{x}\right)$ into (A.7c), we find that $k_{x}$ is uniquely determined by the parameters of the model.

We complete this part of the proof by noting that the non-negativity constraints on the capital goods are not binding in either steady state, because $x_{i}=\delta_{i} k_{i}$ is strictly positive for $\delta_{i} \in(0,1)$. This justifies the above assumption that all first-order conditions hold with equality at the steady state. This also implies that there will be neighborhood of the steady state in which all first-order conditions hold with equality.

\section{A.2 Linear $f$}

\section{A.2.1 Reduced-form dynamics}

Assuming interior solutions and following the same steps as before, one can show that with a linear $f$ the equilibrium dynamics are characterized by the following equations:

$$
\begin{aligned}
& \dot{k}_{c t}=x_{c t}-\delta_{c} k_{c t}, \quad \dot{k}_{x t}=x_{x t}-\delta_{x} k_{x t}, \quad k_{x t}^{\beta_{1}} \beta_{x t}^{\beta_{2}}=f_{c} x_{c t}+f_{x} x_{x t}, \\
& l_{c t}=(1-a), \quad l_{x t}=\left[\frac{(1-b) \mu_{x t}}{f_{x}}\right]^{\frac{1}{1-\beta_{2}}} k_{x t}^{\frac{\beta_{1}}{1-\beta_{2}},}, \\
& \frac{\mu_{c t}}{\mu_{x t}}=\frac{f_{c}}{f_{x}}, \quad \dot{\mu}_{c t}=\mu_{c t}\left(\rho+\delta_{c}\right)-\frac{a}{1-a} \frac{l_{c t}}{k_{c t}}, \quad \dot{\mu}_{x t}=\mu_{x t}\left(\rho+\delta_{x}\right)-\frac{b}{1-b} \frac{l_{x t}}{k_{x t}} .
\end{aligned}
$$


If none of the non-negativity constraints on $x_{c t}$ and $x_{x t}$ binds, then we can reduce these equations to three equations in $k_{c t}, k_{x t}$, and $\mu_{c t}$ that describe the reduced-form equilibrium dynamics:

$$
\begin{aligned}
& f_{c} \dot{k}_{c t}+f_{x} \dot{k}_{x t}=\left[\frac{(1-b) \mu_{c t}}{f_{c}}\right]^{\frac{\beta_{2}}{1-\beta_{2}}} k_{x t}^{\frac{\beta_{1}}{1-\beta_{2}}}-f_{c} \delta_{c} k_{c t}-f_{x} \delta_{x} k_{x t}, \\
& \dot{\mu}_{c t}=\mu_{c t}\left(\rho+\delta_{c}\right)-\frac{a}{k_{c t}} \\
& 0=f_{x} \mu_{c t}\left(\delta_{x}-\delta_{c}\right)+\frac{a f_{x}}{k_{c t}}-\frac{f_{c} b}{1-b}\left[\frac{(1-b) \mu_{c t}}{f_{c}}\right]^{\frac{1}{1-\beta_{2}}}{\frac{\beta_{x t}+\beta_{2}-1}{1-\beta_{2}}}^{\frac{\beta_{x t}}{10}}
\end{aligned}
$$

Note that unlike for a strictly quasi-convex $f$, we cannot analytically reduce these three equations to two equations that characterize fully the reduced-form equilibrium dynamics.

\section{A.2.2 Existence and uniqueness of steady state}

In steady state, the three equations in (A.10) become:

$$
\begin{aligned}
& 0=\left[\frac{(1-b) \mu_{c}}{f_{c}}\right]^{\frac{\beta_{2}}{1-\beta_{2}}} k_{x}^{\frac{\beta_{1}}{1-\beta_{2}}}-f_{c} \delta_{c} k_{c}-f_{x} \delta_{x} k_{x}, \\
& 0=\mu_{c}\left(\rho+\delta_{c}\right)-\frac{a}{k_{c}}, \\
& 0=f_{x} \mu_{c}\left(\delta_{x}-\delta_{c}\right)+\frac{a f_{x}}{k_{c}}-\frac{f_{c} b}{1-b}\left[\frac{(1-b) \mu_{c}}{f_{c}}\right]^{\frac{1}{1-\beta_{2}}} k_{x}^{\frac{\beta_{1}+\beta_{2}-1}{1-\beta_{2}}} .
\end{aligned}
$$

The existence and uniqueness of the steady state can be shown as follows. First, (A.11b) implies that $k_{c}$ is a function of $\mu_{c}$. Second, substituting the result into (A.11c) implies that $k_{x}$ too is a function of $\mu_{c}$. Third, substituting these two expressions into (A.11a) and rearranging gives the steady state value for $\mu_{c}$. Finally, (A.9) shows that all other steady state variables are functions of $k_{c}, k_{x}$, and $\mu_{c}$.

We complete the proof by noting that the non-negativity constraints on the capital goods are not binding in either steady state, because $x_{i}=\delta_{i} k_{i}$ is strictly positive for $\delta_{i} \in(0,1)$. This justifies the above assumption that all first-order conditions hold with equality at the steady state. This also implies that there will be neighborhood of the steady state in which all first-order conditions hold with equality. 


\section{B Proof of Proposition 2}

\section{B.1 Linear $f$}

\section{B.1.1 Computation of the determinant and the trace}

We start with the linearization of (A.10) at the steady state:

$$
\begin{aligned}
\dot{k}_{t}= & \frac{\beta_{2}}{1-\beta_{2}} \frac{f_{c} \delta_{c} k_{c}+f_{x} \delta_{x} k_{x}}{\mu_{c}}\left(\mu_{c t}-\mu_{c}\right)+\left[\frac{\beta_{1}}{1-\beta_{2}} \frac{f_{c} \delta_{c} k_{c}+f_{x} \delta_{x} k_{x}}{k_{x}}-f_{x}\left(\delta_{x}-\delta_{c}\right)\right]\left(k_{x t}-k_{x}\right)-\delta_{c}\left(k_{t}-k\right), \\
\dot{\mu}_{c t}= & \left(\rho+\delta_{c}\right)\left(\mu_{c t}-\mu_{c}\right)-\frac{f_{x}\left(\rho+\delta_{c}\right) \mu_{c}}{f_{c} k_{c}}\left(k_{x t}-k_{x}\right)+\frac{\left(\rho+\delta_{x}\right) \mu_{c}}{f_{c} k_{c}}\left(k_{t}-k\right), \\
0= & {\left[-\left(\rho+\delta_{c}\right)+\left(\rho+\delta_{x}\right)-\frac{1}{1-\beta_{2}}\left(\rho+\delta_{x}\right)\right]\left(\mu_{c t}-\mu_{c}\right) } \\
& +\left[\frac{f_{x}\left(\rho+\delta_{c}\right) \mu_{c}}{f_{c} k_{c}}-\frac{\beta_{1}+\beta_{2}-1}{1-\beta_{2}} \frac{\left(\rho+\delta_{x}\right) \mu_{c}}{k_{x}}\right]\left(k_{x t}-k_{x}\right)+\frac{\left(\rho+\delta_{c}\right) \mu_{c}}{f_{c} k_{c}}\left(k_{t}-k\right)
\end{aligned}
$$

where $k_{t} \equiv f_{c} k_{c}+f_{x} k_{x}$. Rearranging gives:

$$
\begin{aligned}
\dot{k}_{t}= & \frac{\beta_{2}}{1-\beta_{2}} \frac{\rho+\delta_{x}}{b} \frac{f_{x} k_{x}}{\mu_{c}}\left(\mu_{c t}-\mu_{c}\right)+\left[\frac{\beta_{1}}{1-\beta_{2}} \frac{\rho+\delta_{x}}{b}-\left(\delta_{x}-\delta_{c}\right)\right] f_{x}\left(k_{x t}-k_{x}\right)-\delta_{c}\left(k_{t}-k\right), \\
\dot{\mu}_{c t}= & \left(\rho+\delta_{c}\right)\left(\mu_{c t}-\mu_{c}\right)-\frac{\left(\rho+\delta_{c}\right)\left[\rho+(1-b) \delta_{x}\right]}{\delta_{x} b} \frac{\mu_{c}}{k_{x}}\left(k_{x t}-k_{x}\right)+\frac{\left(\rho+\delta_{x}\right) \delta_{c} b}{\rho+(1-b) \delta_{x}} \frac{\mu_{c}}{f_{x} k_{x}}\left(k_{t}-k\right), \\
0= & -\left[\left(\rho+\delta_{c}\right)+\frac{\beta_{2}}{1-\beta_{2}}\left(\rho+\delta_{x}\right)\right]\left(\mu_{c t}-\mu_{c}\right) \\
& +\left[\frac{f_{x}\left(\rho+\delta_{c}\right) \delta_{c} b}{\rho+(1-b) \delta_{x}}-\frac{\beta_{1}+\beta_{2}-1}{1-\beta_{2}}\left(\rho+\delta_{x}\right)\right] \frac{\mu_{c}}{k_{x}}\left(k_{x t}-k_{x}\right)+\frac{\left(\rho+\delta_{c}\right) \delta_{c} b}{\rho+(1-b) \delta_{x}} \frac{\mu_{c}}{f_{x} k_{x}}\left(k_{t}-k\right) .
\end{aligned}
$$

The last equation can be solved for $k_{x t}-k_{x}$

$$
k_{x t}-k_{x}=\frac{k_{x}}{\mu_{c}} \frac{\left[\left(1-\beta_{2}\right)\left(\rho+\delta_{c}\right)+\beta_{2}\left(\rho+\delta_{x}\right)\right]\left(\mu_{c t}-\mu_{c}\right)+\left(1-\beta_{2}\right) \frac{\delta_{c} b\left(\rho+\delta_{c}\right)}{\rho+(1-b) \delta_{x}} \frac{\mu_{c}}{\frac{b}{x} k_{x}}\left(k_{t}-k\right)}{\left(1-\beta_{2}\right) \frac{b \delta_{c}\left(\rho+\delta_{c}\right)}{\rho+(1-b) \delta_{x}}-\left(\rho+\delta_{x}\right)\left(\beta_{1}+\beta_{2}-1\right)},
$$

Substituting this back to the two dynamic equations leads to

$$
\left[\begin{array}{c}
\dot{k}_{t} \\
\dot{\mu}_{c t}
\end{array}\right]=\left[\begin{array}{ll}
a_{11} & a_{12} \\
a_{21} & a_{22}
\end{array}\right]\left[\begin{array}{c}
k_{t}-k \\
\mu_{c t}-\mu_{c}
\end{array}\right]
$$


where

$$
\begin{aligned}
& a_{11}=\frac{\frac{\left(\rho+\delta_{c}\right)\left(\beta_{1}\left(\rho+\delta_{x}\right)-\left(1-\beta_{2}\right) b \delta_{x}\right)}{\rho+(1-b) \delta_{x}}+\left(\rho+\delta_{x}\right)\left(\beta_{1}+\beta_{2}-1\right)}{\left(1-\beta_{2}\right) \frac{b \delta_{c}\left(\rho+\delta_{c}\right)}{\rho+(1-b) \delta_{x}}-\left(\rho+\delta_{x}\right)\left(\beta_{1}+\beta_{2}-1\right)}, \\
& a_{12}=\left\{\frac{\beta_{2}}{1-\beta_{2}} \frac{\rho+\delta_{x}}{b}+\frac{\left[\frac{\beta_{1}}{1-\beta_{2}} \frac{\rho+\delta_{x}}{b}+\left(\delta_{c}-\delta_{x}\right)\right]\left[\left(\rho+\delta_{c}\right)-\beta_{2}\left(\delta_{c}-\delta_{x}\right)\right]}{\left(1-\beta_{2}\right) \frac{b \delta_{c}\left(\rho+\delta_{c}\right)}{\rho+(1-b) \delta_{x}}-\left(\rho+\delta_{x}\right)\left(\beta_{1}+\beta_{2}-1\right)}\right\} \frac{f_{x} k_{x}}{\mu_{c}}, \\
& a_{21}=-\frac{\left(\beta_{1}+\beta_{2}-1\right) b\left(\rho+\delta_{c}\right) \frac{\rho+\delta_{x}}{\rho+(1-b) \delta_{x}}}{\left(1-\beta_{2}\right) \frac{b \delta_{c}\left(\rho+\delta_{c}\right)}{\rho+(1-b) \delta_{x}}-\left(\rho+\delta_{x}\right)\left(\beta_{1}+\beta_{2}-1\right)} \frac{\mu_{c}}{f_{x} k_{x}}, \\
& a_{22}=-\frac{\left(\rho+\delta_{c}\right)\left[\frac{b \delta_{c}\left(\rho+\delta_{x}\right)}{\rho+(1-b) \delta_{x}}+\left(\rho+\delta_{x}\right)\left(\beta_{1}+\beta_{2}-1\right)\right]}{\left(1-\beta_{2}\right) \frac{b \delta_{c}\left(\rho+\delta_{c}\right)}{\rho+(1-b) \delta_{x}}-\left(\rho+\delta_{x}\right)\left(\beta_{1}+\beta_{2}-1\right)}
\end{aligned}
$$

The determinant and the trace of the matrix in (B.1) are found to be:

$$
\begin{aligned}
\text { Det } & =\frac{\delta_{c}\left(\rho+\delta_{c}\right)\left[\rho+(1-b) \delta_{x}\right]\left(1-\beta_{1}\right)}{\left(\rho+\delta_{x}\right)\left[\rho+(1-b) \delta_{x}\right]\left(\beta_{1}+\beta_{2}-1\right)-b \delta_{c}\left(\delta_{c}+\rho\right)\left(1-\beta_{2}\right)} \\
\operatorname{Tr} & =\frac{\rho\left(\rho+\delta_{x}\right)\left[\rho+(1-b) \delta_{x}\right]\left(\beta_{1}+\beta_{2}-1\right)+\delta_{c}\left(\delta_{c}+\rho\right)\left[b\left(\delta_{x}+\rho \beta_{2}\right)-\beta_{1}\left(\rho+\delta_{x}\right)\right]}{\left(\rho+\delta_{x}\right)\left[\rho+(1-b) \delta_{x}\right]\left(\beta_{1}+\beta_{2}-1\right)-b \delta_{c}\left(\delta_{c}+\rho\right)\left(1-\beta_{2}\right)} .
\end{aligned}
$$

\section{B.1.2 Characterization of the stability properties}

The steady state is saddle-path stable if Det $<0$, it is stable if $\operatorname{Tr}<0<$ Det, and it is unstable if Tr, Det $>0$. In order to characterize the different cases, first note that the denominators of the trace and the determinant are the same. Second, the numerator of the determinant is always positive. So the local stability properties will depend only on the signs of the numerator of the trace and on the common denominator. Through $\beta_{1}$ and $\beta_{2}$ they both depend on $\theta_{x}$, so we will write $N\left(\theta_{x}\right)$ and $D\left(\theta_{x}\right)$. To find their signs, we first find the values of $\theta_{x}$ for which they become zero:

$$
\begin{aligned}
& D\left(\underline{\theta}_{x}\right)=0 \Longleftrightarrow \underline{\theta}_{x}=\frac{b^{2} \delta_{c}\left(\rho+\delta_{c}\right)}{\left(\rho+\delta_{x}\right)\left[\rho+(1-b) \delta_{x}\right]+(1-b) b \delta_{c}\left(\rho+\delta_{c}\right)} \\
& N\left(\bar{\theta}_{x}\right)=0 \Longleftrightarrow \bar{\theta}_{x}=\frac{b^{2} \delta_{c}\left(\rho+\delta_{c}\right)}{\left(\rho+\delta_{x}\right)\left[\rho+(1-b) \delta_{x}\right]-\frac{\rho b+\delta_{x}}{\rho} b \delta_{c}\left(\rho+\delta_{c}\right)}
\end{aligned}
$$


We can see that $D\left(\theta_{x}\right)<0$ if and only if $\theta_{x}<\underline{\theta}_{x}, D\left(\theta_{x}\right)>0$ if and only if $\theta_{x}>\underline{\theta}_{x}, N\left(\theta_{x}\right)<0$ if and only if $\theta_{x}<\bar{\theta}_{x}$, and $N\left(\theta_{x}\right)>0$ if and only if $\theta_{x}>\bar{\theta}_{x}$. Now, if the condition in (i.a) holds then $0<\underline{\theta}_{x}<\bar{\theta}_{x}$ and if the condition in (i.b) holds then $\underline{\theta}_{x}<0<\bar{\theta}_{x}$. Using this to determine the signs of the determinant and the trace proves our claims.

\section{B.2 Strictly quasi-convex $f$}

\section{B.2.1 Computation of the determinant and the trace}

We again represent the steady values of $f, g$, and their derivatives by dropping their arguments, so $f \equiv f\left(\frac{x_{c}}{x_{x}}, 1\right), g \equiv g\left(\frac{x_{c}}{x_{x}}\right)$, etc. We start the proof by listing some helpful identities that have to hold in our model. First, the definition of $g$ as the inverse of $\frac{f_{c}}{f_{x}}$ implies that

$$
g^{\prime}=\frac{f_{x}^{2}}{f_{c c} f_{x}-f_{c} f_{x c}}
$$

Second, the linear homogeneity of $f$ implies:

$$
f=g f_{c}+f_{x}, \quad 0=g f_{c c}+f_{c x}, \quad 0=f_{x x}+g f_{c x} .
$$

Third, (A.6) and (B.5b) give

$$
\frac{\rho+\delta_{x}(1-b)}{b \delta_{x}}=\frac{g f_{c}}{f_{x}}, \quad \frac{\rho+\delta_{x}(1-b)}{\rho+\delta_{x}}=\frac{g f_{c}}{f},
$$

Finally, using this and (B.5a), we find:

$$
\frac{f_{x c}}{f_{x}} g^{\prime} \frac{\mu_{c}}{\mu_{x}}=\frac{f_{x c} f_{c}}{f_{c c} f_{x}-f_{c} f_{x c}}=-\frac{g f_{c}}{f_{x}+g f_{c}}=-\frac{\rho+\delta_{x}(1-b)}{\rho+\delta_{x}}
$$

The first step of the derivation of the determinant and the trace is to linearize the reduced-form dynamics at the steady state. Indicating steady state variables by dropping the time subscript, the result 
is:

$$
\left[\begin{array}{l}
\dot{k}_{c t} \\
\dot{k}_{x t} \\
\dot{\mu}_{c t} \\
\dot{\mu}_{x t}
\end{array}\right]=\left[\begin{array}{llll}
a_{11} & a_{12} & a_{13} & a_{14} \\
a_{21} & a_{22} & a_{23} & a_{24} \\
a_{31} & a_{32} & a_{33} & a_{34} \\
a_{41} & a_{42} & a_{43} & a_{44}
\end{array}\right]\left[\begin{array}{l}
k_{c t}-k_{c} \\
k_{x t}-k_{x} \\
\mu_{c t}-\mu_{c} \\
\mu_{x t}-\mu_{x}
\end{array}\right],
$$

where: ${ }^{16}$

$$
\begin{aligned}
& a_{11}=-\delta_{c}, \quad a_{12}=\frac{\beta_{1}}{1-\beta_{2}} \frac{\delta_{c} k_{c}}{k_{x}}, \quad a_{13}=\left[\frac{g^{\prime}}{g} \frac{\mu_{c}}{\mu_{x}}-\frac{\beta_{2}}{1-\beta_{2}} \frac{f_{x c}}{f_{x}} g^{\prime} \frac{\mu_{c}}{\mu_{x}}-\frac{g f_{c}}{f} \frac{g^{\prime}}{g} \frac{\mu_{c}}{\mu_{x}}\right] \frac{\delta_{c} k_{c}}{\mu_{c}}, \\
& a_{14}=\left[\frac{\beta_{2}}{1-\beta_{2}}-\frac{g^{\prime}}{g} \frac{\mu_{c}}{\mu_{x}}+\frac{\beta_{2}}{1-\beta_{2}} \frac{f_{x c}}{f_{x}} g^{\prime} \frac{\mu_{c}}{\mu_{x}}+\frac{f_{c}}{f} g^{\prime} \frac{\mu_{c}}{\mu_{x}}\right] \frac{\delta_{c} k_{c}}{\mu_{x}}, \quad a_{21}=0, \quad a_{22}=\frac{\beta_{1}}{1-\beta_{2}} \frac{\delta_{x} k_{x}}{k_{x}}-\delta_{x}, \\
& a_{23}=\left[\frac{\beta_{2}}{1-\beta_{2}} \frac{f_{x c}}{f_{x}} g^{\prime} \frac{\mu_{c}}{\mu_{x}}-\frac{f_{c}}{f} g^{\prime} \frac{\mu_{c}}{\mu_{x}}\right] \frac{\delta_{x} k_{x}}{\mu_{c}}, \quad a_{24}=\left[\frac{\beta_{2}}{1-\beta_{2}}-\frac{\beta_{2}}{1-\beta_{2}} \frac{f_{x c}}{f_{x}} g^{\prime} \frac{\mu_{c}}{\mu_{x}}+\frac{g f_{c}}{f} \frac{g^{\prime}}{\left.\frac{\mu_{c}}{\mu_{x}}\right]} \frac{\delta_{x} k_{x}}{\mu_{x}},\right. \\
& a_{31}=\frac{\left(\rho+\delta_{c}\right) \mu_{c}}{k_{c}}, \quad a_{32}=0, \quad a_{33}=\rho+\delta_{c}, \quad a_{34}=0, \\
& a_{41}=0, \quad a_{42}=\frac{\beta_{1}+\beta_{2}-1}{1-\beta_{2}} \frac{\left(\rho+\delta_{x}\right) \mu_{x}}{k_{x}}, \quad a_{43}=\frac{1}{1-\beta_{2}} \frac{f_{x c}}{f_{x}} g^{\prime} \frac{\mu_{c}}{\mu_{x}} \frac{\left(\rho+\delta_{x}\right) \mu_{x}}{\mu_{c}}, \\
& a_{44}=\left(\rho+\delta_{x}\right)-\frac{1}{1-\beta_{2}}\left(\rho+\delta_{x}\right)-\frac{1}{1-\beta_{2}}\left(\rho+\delta_{x}\right) \frac{f_{x c}}{f_{x}} g^{\prime} \frac{\mu_{c}}{\mu_{x}} .
\end{aligned}
$$

To simplify these expressions, it is useful to define the elasticity of the investment ratio with respect to the relative price evaluated at the steady state. Denoting the inverse of that elasticity by $\varepsilon \geq 0,{ }^{17}$ we have:

$$
\varepsilon \equiv \frac{g\left(\frac{\mu_{c}}{\mu_{x}}, 1\right)}{g^{\prime}\left(\frac{\mu_{c}}{\mu_{x}}, 1\right)} \frac{1}{\frac{\mu_{c}}{\mu_{x}}} .
$$

Now, using (B.6a) and (B.6b), the previous terms can be rewritten:

$$
\begin{aligned}
& a_{11}=-\delta_{c}, \quad a_{12}=\frac{\beta_{1}}{1-\beta_{2}} \frac{\delta_{c} k_{c}}{k_{x}}, \quad a_{13}=\left[\frac{\beta_{2}}{1-\beta_{2}}+\frac{1}{\varepsilon} \frac{1-(1+\varepsilon) \beta_{2}}{1-\beta_{2}} \frac{\delta_{x} b}{\rho+\delta_{x}}\right] \frac{\delta_{c} k_{c}}{\mu_{c}}, \\
& a_{14}=-\frac{1}{\varepsilon} \frac{1-(1+\varepsilon) \beta_{2}}{1-\beta_{2}} \frac{\delta_{c} k_{c}}{\mu_{x}} \frac{\delta_{x} b}{\rho+\delta_{x}}, \quad a_{21}=0, \quad a_{22}=\delta_{x} \frac{\beta_{1}+\beta_{2}-1}{1-\beta_{2}},
\end{aligned}
$$

\footnotetext{
${ }^{16}$ To find these expressions we have repeatedly used the fact that if a function is of the form $h\left(x_{1}, x_{2}, x_{3}\right)=x_{1}^{\alpha} x_{2}^{\beta}-a x_{3}$, then its partial derivative can be written as $\frac{\partial h}{\partial x_{1}}=\alpha \frac{f\left(x_{1}, x_{2}, x_{3}\right)+a x_{3}}{x_{1}}$.

${ }^{17}$ Note that if $f$ is parameterized by $\varepsilon$ according to (6), then the inverse elasticity in the case is also given by $\varepsilon$.
} 


$$
\begin{aligned}
& a_{23}=-\frac{1}{\varepsilon} \frac{1-(1+\varepsilon) \beta_{2}}{1-\beta_{2}} \frac{\delta_{x} k_{x}}{\mu_{c}} \frac{\rho+\delta_{x}(1-b)}{\rho+\delta_{x}}, \quad a_{24}=\left[\frac{\beta_{2}}{1-\beta_{2}}+\frac{1}{\varepsilon} \frac{1-(1+\varepsilon) \beta_{2}}{1-\beta_{2}} \frac{\rho+\delta_{x}(1-b)}{\rho+\delta_{x}}\right] \frac{\delta_{x} k_{x}}{\mu_{x}}, \\
& a_{31}=\frac{\left(\rho+\delta_{c}\right) \mu_{c}}{k_{c}}, \quad a_{32}=0, \quad a_{33}=\rho+\delta_{c}, \quad a_{34}=a_{41}=0, \\
& a_{42}=-\frac{\beta_{1}+\beta_{2}-1}{1-\beta_{2}} \frac{\mu_{x}}{k_{x}}\left(\rho+\delta_{x}\right), \quad a_{43}=-\frac{1}{1-\beta_{2}}\left[\rho+\delta_{x}(1-b)\right] \frac{\mu_{x}}{\mu_{c}}, \quad a_{44}=\left(\rho+\delta_{x}\right)-\frac{1}{1-\beta_{2}} \delta_{x} b .
\end{aligned}
$$

The second step is to combined the terms just derived and actually compute the determinant and the trace. Using the fact that $a_{32}=a_{34}=a_{41}=0$, the determinant can be written as

$$
\begin{aligned}
\text { Det } & =a_{31} a_{42}\left(a_{13} a_{24}-a_{14} a_{23}\right)+a_{22} a_{31}\left(a_{14} a_{43}-a_{13} a_{44}\right) \\
& +a_{11} a_{33}\left(a_{22} a_{44}-a_{24} a_{42}\right)+a_{12} a_{31}\left(a_{23} a_{44}-a_{24} a_{43}\right) .
\end{aligned}
$$

Using the previous expressions, the four terms in that determinant are found to equal:

$$
\begin{aligned}
& a_{31} a_{42}\left(a_{13} a_{24}-a_{14} a_{23}\right)=-\frac{1}{\varepsilon} \frac{\beta_{2}}{1-\beta_{2}} \frac{\beta_{1}+\beta_{2}-1}{1-\beta_{2}} \delta_{c} \delta_{x}\left(\rho+\delta_{c}\right)\left(\rho+\delta_{x}\right), \\
& a_{22} a_{31}\left(a_{14} a_{43}-a_{13} a_{44}\right)=\frac{\beta_{1}+\beta_{2}-1}{1-\beta_{2}} \frac{\beta_{2}}{1-\beta_{2}} \frac{(1+\varepsilon) \delta_{x} b-\varepsilon\left(\rho+\delta_{x}\right)}{\varepsilon} \delta_{c} \delta_{x}\left(\rho+\delta_{c}\right), \\
& a_{11} a_{33}\left(a_{22} a_{44}-a_{24} a_{42}\right)=-\frac{\beta_{1}+\beta_{2}-1}{1-\beta_{2}} \frac{1+\varepsilon}{\varepsilon} \delta_{x} \delta_{c}\left(\rho+\delta_{c}\right)\left[\rho+\delta_{x}(1-b)\right], \\
& a_{12} a_{31}\left(a_{23} a_{44}-a_{24} a_{43}\right)=\frac{\beta_{1}}{1-\beta_{2}} \frac{\beta_{2}}{1-\beta_{2}} \frac{1+\varepsilon}{\varepsilon} \delta_{c} \delta_{x}\left(\rho+\delta_{c}\right)\left[\rho+\delta_{x}(1-b)\right] .
\end{aligned}
$$

Using these expressions and simplifying, we find the determinant:

$$
\text { Det }=\frac{1+\varepsilon}{\varepsilon} \frac{\delta_{c} \delta_{x}\left(\rho+\delta_{c}\right)\left[\rho+\delta_{x}(1-b)\right]\left(1-\beta_{1}\right)}{1-\beta_{2}} .
$$

In general form the trace is given by:

$$
\operatorname{Tr}=a_{11}+a_{22}+a_{33}+a_{44}
$$


Substituting in the previous expressions for $a_{i i}$, we find the trace:

$$
\operatorname{Tr}=2 \rho+\delta_{x} \frac{\beta_{1}-b}{1-\beta_{2}} .
$$

\section{B.2.2 Characterization of the stability properties}

We start with the case $\theta_{x} \in\left[0, \frac{b}{1-b}\right)$, implying that $\beta_{2}<1$. Then Det $>0$ and $\operatorname{Tr}>0 .{ }^{18}$ Now suppose that the steady state were stable. Then (B.7) would have three or four eigenvalues with negative real parts. If (B.7) had four eigenvalues with negative real parts, then the trace would have to be negative, which is a contraction. If (B.7) had three eigenvalues with negative real part, then the determinant would have to be negative, which is a contradiction.

We continue with the case $\theta_{x} \in\left[\frac{b}{1-b}, \frac{1-b}{b}\right)$, implying that $\beta_{2}>1$. Then Det $<0$. Suppose that the steady state were saddle-path stable. Then (B.7) would have two eigenvalues with negative real part and two eigenvalues with positive real part. Irrespective of whether they are real or complex conjugates, this would imply that the determinant must become positive, which is a contraction.

\section{Proof of Proposition 3}

The proof of this proposition follows because using $\frac{\mu_{x}}{\mu_{c}}=\frac{f_{x}}{f_{c}}$, one can show that the limits of the steady state versions of the four equations in (A.4), which characterize uniquely the steady state with quasiconvex $f$, imply the three equations in (A.11), which characterize uniquely the steady state with linear $f$. In particular, $f_{c}$ times (A.4c) plus $f_{x}$ times (A.4d) converges to (A.11a). Second, (A.4a) is identical to equation (A.11b). Third, $f_{c}$ times (A.4b) minus $f_{x}$ times (A.4a) converges to (A.11c).

\footnotetext{
${ }^{18}$ Recall that $\beta_{1}=\left(1+\theta_{x}\right) b$, so $\beta_{1}-b=\theta_{x} b \geq 0$.
} 


\section{Proof of Proposition 4}

We start by defining the production possibility frontier between the consumption good, $c_{t}$, and the composite capital good, $x_{t} \equiv f\left(x_{c t}, x_{x t}\right)$ :

$$
\begin{array}{ll} 
& \max _{x_{c t-\Delta t}, x_{x t-\Delta t} l_{x t}, l_{c t}} x_{t}\left(c_{t}\right) \\
\text { s.t. } & x_{t} \leq k_{x t}^{\beta_{1}} l_{x t}^{\beta_{2}}, \quad c_{t} \leq k_{c t}^{\alpha_{1}} l_{c t}^{\alpha_{2}}, \quad l_{c t}+l_{x t} \leq \bar{l}_{t}, \\
& k_{c t} \leq\left(x_{c t-\Delta t}-\delta_{c} \bar{k}_{c t-\Delta t}\right) \Delta t+\bar{k}_{c t-\Delta t}, \quad k_{x t} \leq\left(x_{x t-\Delta t}-\Delta_{x} \bar{k}_{x t-\Delta t}\right) \Delta t+\bar{k}_{x t-\Delta t}, \\
& f\left(x_{c t-\Delta t}, x_{x t-\Delta t}\right) \leq \bar{x}_{t-\Delta t},
\end{array}
$$

where $\bar{l}_{t}, \bar{k}_{c t-\Delta t}, \bar{k}_{x t-\Delta t}, \bar{x}_{t-\Delta t}$ are given. The solution to this problem determines for given feasible $c_{t}$ the maximal level of $x_{t}$. We use $\Delta t$ in writing this problem because of the sector-specificity of capital, which means that at some time $t-\Delta t, \Delta$ being small, the two new capital goods need to be chosen.

Now rewrite the problem as:

$$
\begin{aligned}
\max _{x_{c t-\Delta t}, x_{x t-\Delta t}, l_{x t}} & {\left[\left(x_{x t-\Delta t}-\delta_{x} \bar{k}_{x t-\Delta t}\right) \Delta t+\bar{k}_{x t-\Delta t}\right]^{\beta_{1}} l_{x t}^{\beta_{2}} } \\
\text { s.t. } \quad c_{t} & =\left[\left(x_{c t-\Delta t}-\delta_{c} \bar{k}_{c t-\Delta t}\right) \Delta t+\bar{k}_{c t-\Delta t}\right]^{\alpha_{1}}\left[\bar{l}_{t}-l_{x t}\right]^{\alpha_{2}}, \\
\bar{x}_{t-\Delta t} & =f\left(x_{c t-\Delta t}, x_{x t-\Delta t}\right) .
\end{aligned}
$$

The necessary first-order conditions are:

$$
\begin{aligned}
& \left.x_{t}=\left[x_{x t-\Delta t}-\delta_{x} \bar{k}_{x t-\Delta t}\right) \Delta t+\bar{k}_{x t-\Delta t}\right]^{\beta_{1}} l_{x t-\Delta t}^{\beta_{2}} \\
& c_{t}=\left[\left(x_{c t-\Delta t}-\delta_{c} \bar{k}_{c t-\Delta t}\right) \Delta t+\bar{k}_{c t-\Delta t}\right]^{\alpha_{1}}\left[\bar{l}_{t}-l_{x t}\right]^{\alpha_{2}} \\
& \frac{b}{1-b} \frac{l_{x t}}{\left(x_{x t-\Delta t}-\bar{x}_{x} \bar{x}_{x t-\Delta t}\right) \Delta t+\bar{k}_{x t-\Delta t}} \frac{f_{c}\left(x_{c t-\Delta t}, x_{x t-\Delta t}\right)}{f_{x}\left(x_{c t-\Delta t}, x_{x t-\Delta t}\right)}=\frac{a}{1-a} \frac{\bar{l}_{-} l_{x t}}{\left(x_{c t-\Delta t}-\bar{k}_{c t-\Delta t}\right) \Delta t+\bar{k}_{c t-\Delta t}}, \\
& \bar{x}_{t-\Delta t}=f\left(x_{c t-\Delta t}, x_{x t-\Delta t}\right) .
\end{aligned}
$$

These four equations define the production possibility frontier between $c_{t}$ and $x_{t}$. 
Inspecting the optimization problem in (D.2) for $f\left(x_{c t}, x_{x t}\right)=f_{c} x_{c t}+f_{x} x_{x t}$, we can see that the constraint (D.2c) becomes

$$
\bar{x}_{t-\Delta t}=f_{c} x_{c t-\Delta t}+f_{x} x_{x t-\Delta t} .
$$

Therefore the first-order conditions are (D.3a) and (D.3b) as before and

$$
\begin{aligned}
\frac{b}{1-b} \frac{l_{x t+\Delta t}}{\left(x_{x t-\Delta t}-\delta_{x} k_{x t-\Delta t}\right) \Delta t+k_{x t-\Delta t}} \frac{f_{c}}{f_{x}} & =\frac{a}{1-a} \frac{\bar{l}_{t+\Delta t}-l_{x t+\Delta t}}{\left(x_{c t-\Delta t}-\delta_{c} k_{c t-\Delta t}\right) \Delta t+k_{c t-\Delta t}} . \\
f_{c} x_{c t-\Delta t}+f_{x} x_{x t-\Delta t} & =\bar{x}_{t-\Delta t} .
\end{aligned}
$$

(D.3c) and (D.3d) converge to (D.3c') and (D.3d') as $f_{i} \rightarrow f$ in $U\left(x_{c}, x_{x}\right)$.

\section{References}

Aiyagari, S. Rao, “Comments on Farmer and Guo's “The Econometrics of Indeterminacy: An Applied Study”," Carnegie Rochester Conference Series on Public Policy, 1995, 43.

Bartelsman, Eric, Ricardo Caballero, and Richard K. Lyons, "Consumer and Supplier Driven Externalities," American Economic Review, 1994, 84, 1075-1084.

Basu, Susanto and John G. Fernald, "Returns to Scale in U.S. Production: Estimates and Implications," Journal of Political Economy, 1997, 105, 249-283.

Benhabib, Jess and Kazuo Nishimura, "Indeterminacy and Constant Returns," Journal of Economic Theory, 1998, 81, 58-96.

— and Roger E. A. Farmer, "Indeterminacy and Increasing Returns," Journal of Economic Theory, 1994, 63, 19-41.

— and — - "Indeterminacy and Sector-Specific Externalities," Journal of Monetary Economics, 1996, 37, 421-443. 
— and — , "Indeterminacy and Sunspots in Macroeconomics," in John B. Taylor and Michael Woodford, eds., Handbook of Macroeconomics, Amsterdam: North-Holland, 1999.

—, Qinglai Meng, and Kazuo Nishimura, "Indeterminacy under Constant Returns to Scale in Multisector Economies," Econometrica, 2000, 68, 1541-1548.

Boldrin, Michele and Aldo Rustichini, "Growth and Indeterminacy in Dynamic Models with Externalities," Econometrica, 1994, 62, 323-342.

—, Lawrence J. Christiano, and Jonas D.M. Fisher, "Habit Persistence, Asset Returns and the Business Cycle," American Economic Review, 2001, 91, 149-166.

Burnside, Craig, "Production Function Regressions, Returns to Scale, and Externalities," Journal of Monetary Economics, 1996, 37, 177-201.

—, Martin Eichenbaum, and Sergio Rebelo, "Capital Utilization and Returns to Scale," in Ben S. Bernanke and Julio J. Rotemberg, eds., NBER Macroeconomics Annual 1995, Cambridge, MA: MIT Press, 1995.

Christiano, Lawrence J., “A Discrete-Time Version of Benhabib-Farmer II,” Manuscript, Northwestern University, Evanston, IL 1995.

and Sharon G. Harrison, "Chaos, Sunspots, and Automatic Stabilizers," Journal of Monetary Economics, 1999, 44, 3-31.

Farmer, Roger E. A. and Jang Ting Guo, "Real Business Cycles and the Animal Spirit Hypothesis," Journal of Economic Theory, 1994, 63, 42-73.

Fisher, Jonas D. M., "Relative Prices, Complementarities, and Comovement among Components of Aggregate Expenditure," Journal of Monetary Economics, 1997, 39, 449-474.

Guo, Jang-Ting and Kevin J. Lansing, "Fiscal Policy, Increasing Returns and Endogenous Fluctuations," Macroeconomic Dynamics, 2002, 6, 633-664. 
Hall, Robert E., "Relation Between Price and Marginal Cost in U.S. Industry," Journal of Political Economy, 1988, 96, 921-947.

Hansen, Gary D., "Indivisible Labor and the Business Cycle," Journal of Monetary Economics, 1985, $16,309-328$.

Harrison, Sharon G., "Indeterminacy in a Model with Sector-Specific Externalities," Journal of Economic Dynamics and Control, 2001, 25, 747-764.

—_, "Returns to Scale and Externalities in the Consumption and Investment Sectors," Review of Economic Dynamics, 2003, 6, 963-976.

and Mark Weder, "Tracing Externalities as Sources of Indeterminacy,” Journal of Economic Dynamics and Control, 2001, 26, 851-867.

Herrendorf, Berthold, Ákos Valentinyi, and Robert Waldmann, "Ruling Out Multiplicity and Indeterminacy: The Role of Heterogeneity," Review of Economic Studies, 2000, 67, 295-307.

— and — - "Determinacy Through Intertemporal Capital Adjustment Costs," forthcoming: Review of Economic Dynamics, 2003.

Huffman, Gregory W. and Mark A. Wynne, "The Role of Intratemporal Adjustment Costs in a Multisector Economy," Journal of Monetary Economics, 1999, 43, 317-350.

Kim, Jinill, "Indeterminacy and Investment Adjustment Cost: An Analytical Result," forthcoming: Macroeconomic Dynamics, 2003.

Lahiri, Amartya, "Growth and Equilibrium Indeterminacy: The Role of Capital Mobility," Economic Theory, 2001, 17, 197-209.

Lucas, Robert E. Jr. and Edward C. Prescott, "Investment under Uncertainty," Econometrica, 1971, 39, 659-681. 
Morris, Stephen and Hyun Song Shin, "Unique Equilibrium in a Model of Self-Fulfilling Currency Attacks," American Economic Review, 1998, 88, 587-597.

Perli, Roberto, "Indeterminacy, Home Production, and the Business Cycle: A Calibrated Analysis," Journal of Monetary Economics, 1998, 41, 105-125.

Ramey, Valerie A. and Matthew D. Shapiro, "Displaced Capital: A Study of Aerospace Plant Closings," Journal of Political Economy, 2001, 109, 958-992.

Rogerson, Richard, "Indivisible Labor, Lotteries, and Equilibrium," Journal of Monetary Economics, 1988, 21, 3-16.

Schmitt-Grohé, Stephanie, "Endogenous Business Cycles and the Dynamics of Output, Hours, and Consumption," American Economic Review, 2000, 90, 1136-1158.

Weder, Mark, "Fickle Consumers, Durable Goods, and Business Cycles," Journal of Economic Theory, 1998, 81, 37-57.

—_, "Animal Spirits, Technology Shocks, and the Business Cycle," Journal of Economic Dynamics and Control, 2000, 24, 273-295.

Wen, Yi, “Indeterminacy, Dynamic Adjustment Costs, and Cycles,” Economics Letters, 1998, 59, 213 216. 